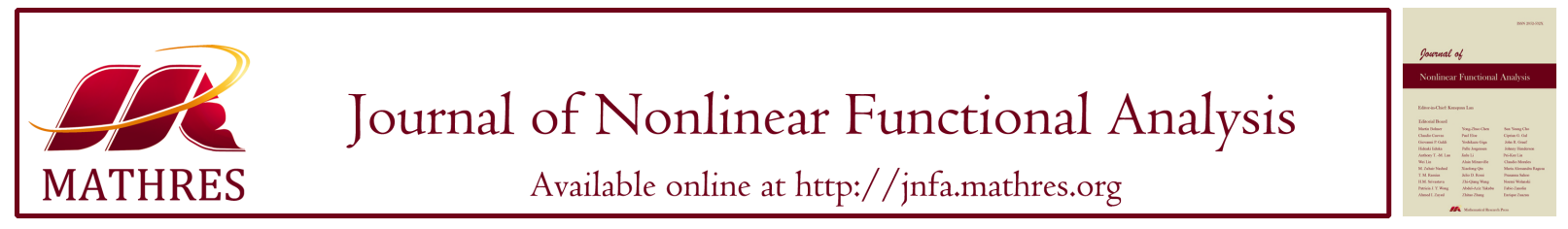

\title{
FIXED POINT THEOREMS FOR CHANDRABHAN TYPE MAPS IN ABSTRACT CONVEX UNIFORM SPACES
}

\author{
HOONJOO KIM \\ Department of Mathematics Education, Sehan University, YoungAm-Gun, Chunnam 58447, Korea
}

\begin{abstract}
The aim of this paper is to present new fixed point theorems for Chandrabhan type multimaps on abstract convex uniform spaces. We obtain fixed point theorems for various Chandrabhan type multimaps such as upper semicontinuous or closed maps in Hausdorff KKM uniform spaces, and the maps whose ranges are $\Phi$-sets. We also obtain fixed point theorems in hyperconvex metric spaces.

Keywords. Abstract convex uniform space; KKM map; Fixed point; Hyperconvex metric space; Upper semicontinuous.
\end{abstract}

\section{INTRODUCTION AND PRELIMINARIES}

Fixed point theorems for multimaps are useful for proving the existence theorems for many problems in differential and integral inclusions, control theory, and optimization; see, e.g., [1, 2, 3] and the references therein. In 1980, Mönch [4] obtained a common generalization of the fixed point theorems of Schauder, Krasnoselskii, Darbo, and Sadovskii. Two decades later, O'Regan and Precup [5] presented the Mönch's result for multimaps in Banach space, and Dhage [6] further generalized Mönch multimaps to Chandrabhan multimaps.

Recently, Amini-Harandi et al. [7] obtained a fixed point theorem for Mönch type multimaps on abstract convex uniform spaces. The concept of abstract convex uniform space was introduced by Park [8] in 2009 as a generalization of locally convex spaces, locally $G$-convex uniform spaces, and other abstract locally convex structures.

The aim of this paper is to present new fixed point theorems for Chandrabhan type multimaps on abstract convex uniform spaces. We obtain fixed point theorems for various Chandrabhan type multimaps such as upper semicontinuous or closed maps in Hausdorff KKM uniform spaces, and the maps whose ranges are $\Phi$-sets. The result extends that of O'Regan and Precup [5] and simplifies that of [7]. Using the result, we obtain fixed point theorems in hyperconvex metric spaces.

\footnotetext{
${ }^{*}$ Corresponding author.

E-mail address: hoonjoo@sehan.ac.kr.

Received February 9, 2021; Accepted May 4, 2021.
} 
A multimap (or simply, a map) $F: X \multimap Y$ is a function from a set $X$ into the power set of $Y$, that is, a function with the values $F(x) \subset Y$ for $x \in X$, and the fibers $F^{-}(y):=\{x \in X \mid y \in F(x)\}$ for $y \in Y$. For $A \subset X$, let $F(A):=\bigcup\{F(x) \mid x \in A\}$. Throughout this paper, we assume that multimaps have nonempty values otherwise explicitly stated or obvious from the context. The closure operation and the interior operation of $F$ are denoted by and $\operatorname{Int} F$, respectively.

Let $\langle X\rangle$ denote the set of all nonempty finite subsets of a set $X$.

From Park $[8,9]$, we have that an abstract convex space $(X, D ; \Gamma)$ consists of a topological space $X$, a non-empty set $D$, and a map $\Gamma:\langle D\rangle \multimap X$ with nonempty values $\Gamma_{A}:=\Gamma(A)$ for $A \in\langle D\rangle$. For any nonempty $D^{\prime} \subset D$, the $\Gamma$-convex hull of $D^{\prime}$ is denoted and defined by $\operatorname{co}_{\Gamma} D^{\prime}:=$ $\bigcup\left\{\Gamma_{A} \mid A \in\left\langle D^{\prime}\right\rangle\right\} \subset X$.

When $D \subset X$ in $(X, D ; \Gamma)$, the space is denoted by $(X \supset D ; \Gamma)$. For $X=D$, let $(X ; \Gamma):=$ $(X, X ; \Gamma)$. When $(X \supset D ; \Gamma)$, a subset $X^{\prime}$ of $X$ is said to be $\Gamma$-convex if $\operatorname{co}_{\Gamma}\left(X^{\prime} \cap D\right) \subset X^{\prime}$. This means that $\left(X^{\prime}, D^{\prime} ; \Gamma^{\prime}\right)$ itself is an abstract convex space where $D^{\prime}:=X^{\prime} \cap D$, and $\Gamma^{\prime}:\left\langle D^{\prime}\right\rangle \multimap X^{\prime}$ is a map defined by $\Gamma_{A}^{\prime}:=\Gamma_{A} \subset X^{\prime}$ for $A \in\left\langle D^{\prime}\right\rangle$.

A generalized convex space or a $G$-convex space $(X, D ; \Gamma)$ consists of a topological space $X$ and a non-empty set $D$ such that, for each $A \in\langle D\rangle$ with the cardinality $|A|=n+1$, there exist a subset $\Gamma_{A}$ of $X$ and a continuous map $\phi_{A}: \Delta_{n} \rightarrow \Gamma_{A}$ such that $J \in\langle A\rangle$ implies $\phi_{A}\left(\Delta_{J}\right) \subset \Gamma_{J}$. Here, $\Delta_{n}$ is the standard $n$-simplex with vertices $\left\{e_{0}\right\}_{i=0}^{n}$, and $\Delta_{J}$ is the face of $\Delta_{n}$ corresponding to $J \in$ $\langle A\rangle$, that is, if $A=\left\{a_{0}, a_{1}, \ldots, a_{n}\right\}$, and $J=\left\{a_{i_{0}}, a_{i_{1}}, \ldots, a_{i_{k}}\right\} \subset A$, then $\Delta_{J}=\operatorname{co}\left\{e_{i_{0}}, e_{i_{1}}, \ldots, e_{i_{k}}\right\}$.

A subset $S$ of $X$ is called a $G$-convex subset of $(X \supset D ; \Gamma)$ if, for any $N \in\langle S \cap D\rangle, \Gamma_{N} \subset S$. For details on $G$-convex spaces, we refer to $[10,11,12]$ and the references therein.

A $c$-space $(X ; \Gamma)$ consists of a topological space $X$, and a map $\Gamma:\langle X\rangle \multimap X$ with nonempty contractible values $\Gamma_{A}:=\Gamma(A)$ for $A \in\langle X\rangle$ satisfying that, for all $A, B \in\langle X\rangle, A \subset B$ implies $\Gamma_{A} \subset \Gamma_{B}$. A nonempty subset $E \subset X$ is an $F$-set if $\Gamma(A) \subset E$ for any $A \in\langle E\rangle$. For details, we refer to Horvath [13]. Note that

$$
\text { a } c \text {-space } \Longrightarrow \text { a } G \text {-convex space } \Longrightarrow \text { an abstract convex space. }
$$

Let $(X ; \Gamma)$ be an abstract convex space, and let $Z$ be a set. For a multimap $F: X \multimap Z$, if a multimap $G: X \multimap Z$ satisfies $F\left(\Gamma_{A}\right) \subset G(A)$ for all $A \in\langle X\rangle$, then $G$ is called a $K K M$ map with respect to $F$. A KKM map $G: X \multimap X$ is a KKM map with respect to the identity map $1_{X}$.

A multimap $F: X \multimap Z$ is called a $\mathfrak{K}$-map if, for a KKM map $G: X \multimap Z$ with respect to $F$, the family $\{G(x)\}_{x \in X}$ has the finite intersection property. The set $\mathfrak{K}(X, Z)$ is defined to be $\{F: X \multimap Z \mid F$ is a $\mathfrak{K}$-map $\}$. Similarly, a $\mathfrak{K} \mathfrak{C}$-map is defined for closed-valued maps $G$ and a $\mathfrak{K O}$-map for open-valued maps $G$.

For an abstract convex space $(X ; \Gamma)$, the $K K M$ principle is the statement $1_{X} \in \mathfrak{K C}(X, X) \cap$ $\mathfrak{K O}(X, X)$. An abstract convex space is called a KKM space if it satisfies the KKM principle. Representative examples of a KKM space are a topological vector space, a $G$-convex space, and a $c$-space. Other examples of KKM spaces are given in [14] and the references therein.

\section{BASIC PROPERTIES OF $L \Gamma$-SPACES}

An abstract convex uniform space $(X, D ; \Gamma ; \mathscr{U})$ is an abstract convex space with a basis $\mathscr{U}$ of a uniform structure of $X$. For $A \subset X$ and $U \in \mathscr{U}$, the set $U[A]$ is defined to be $\{y \in X \mid(x, y) \in$ $U$ for some $x \in A\}$. 
An abstract convex uniform space $(X \supset D ; \Gamma ; \mathscr{U})$ is called an $L \Gamma$-space if $D$ is dense in $X$, and $U[C]$ is $\Gamma$-convex for each $U \in \mathscr{U}$ whenever $C \subset X$ is $\Gamma$-convex. A KKM uniform space $(X ; \Gamma ; \mathscr{U})$ is a KKM space with a basis $\mathscr{U}$ of a uniform structure of $X$.

Let $(X, D ; \Gamma)$ be an abstract convex space and $A \subset X$, and put

$\Gamma-\operatorname{co} A=\bigcap\{C \mid C$ is a $\Gamma$-convex subset of $X$ containing $A\}$, and

$\Gamma-\overline{c o} A=\bigcap\{C \mid C$ is a closed $\Gamma$-convex subset of $X$ containing $A\}$.

Note that $\Gamma-\operatorname{co} A$ and $\Gamma-\overline{\operatorname{co}} A$ are the smallest $\Gamma$-convex set and the smallest closed $\Gamma$-convex set containing $A$, respectively. Clearly, $\operatorname{co}_{\Gamma} A \subset \Gamma-\operatorname{co} A$.

Lemma 2.1. Let $(X \supset D ; \Gamma ; \mathscr{U})$ be an $L \Gamma$-space.

(1) If $C$ is a $\Gamma$-convex subset of $X$, then its closure $\bar{C}$ is $\Gamma$-convex.

(2) If $A \subset X$, then $\Gamma-\overline{\operatorname{co}} A=\overline{\Gamma-\operatorname{co} A \text {. }}$

Proof. (1) If $N \in\langle\bar{C}\rangle$, and $V \in \mathscr{U}$, then $N \subset V(C)$. Since $V(C)$ is $\Gamma$-convex, one has $\Gamma_{N} \subset V(C)$. Hence $\Gamma_{N} \subset \bigcap_{V \in \mathscr{U}} V[C]=\bar{C}$.

(2) Since $\Gamma-\overline{c o} A$ is closed, we have

$$
\overline{\Gamma-\operatorname{co} A} \subset \overline{\Gamma-\overline{\mathrm{co}} A}=\Gamma-\overline{\mathrm{co}} A .
$$

From (1), $\overline{\Gamma-\operatorname{co} A}$ is a closed $\Gamma$-convex set containing $A$, so $\Gamma-\overline{\operatorname{co}} A \subset \overline{\Gamma-\operatorname{co} A}$.

A subset $S$ of a uniform space $X$ is said to be precompact if, for any entourage $V$, there is an $N \in\langle X\rangle$ such that $S \subset V[N]$.

For each $N \in\langle X\rangle, \Gamma$-coN is called a polytope in $X$. An abstract convex uniform space $(X ; \Gamma ; \mathscr{U})$ is called an abstract convex uniform space with precompact polytopes if each polytope in $X$ is precompact.

Lemma 2.2. If $(X \supset D ; \Gamma ; \mathscr{U})$ is an $L \Gamma$-space with precompact polytopes, and $A$ is precompact, then $\Gamma-\operatorname{co} A$ is precompact.

Proof. For any $U \in \mathscr{U}$, we choose $V, W \in \mathscr{U}$ such that $V \circ V \subset U$, and $W \circ W \subset V$. Since $A$ is precompact, and $D$ is dense in $X$, there exist $L \in\langle A\rangle$, and $N \in\langle D\rangle$ such that $A \subset W(L)$, and $L \subset W(N)$.

Hence $A \subset W \circ W(N) \subset V(N) . V(\Gamma$-coN $)$ is a $\Gamma$-convex set containing $V(N)$, so

$$
\Gamma-\operatorname{co} N \subset \Gamma-\operatorname{co} V(N) \subset V(\Gamma-\operatorname{co} N) \text {. }
$$

Since $\Gamma-\operatorname{co} N$ is precompact, there exists an $M \in\langle\Gamma-\operatorname{co} N\rangle$ such that $\Gamma-\operatorname{co} N \subset V(M)$. Therefore

$$
\Gamma-\operatorname{co} A \subset V(\Gamma-\operatorname{co} N) \subset V(V(M)) \subset U(M) .
$$

A $G$-convex uniform space $(X ; \Gamma ; \mathscr{U})$ is a $G$-convex space with a basis $\mathscr{U}$ of a uniform structure of $X$. A $G$-convex uniform space $(X ; \Gamma ; \mathscr{U})$ is said to be an $L G$-space if the uniformity $\mathscr{U}$ has a base $\mathscr{B}$ such that for each $U \in \mathscr{B}, U[C]$ is $\Gamma$-convex for each $U \in \mathscr{U}$ whenever $C \subset X$ is $\Gamma$-convex. The examples of $G$-convex uniform space were given in [15].

A metric space $(X, d ; \Gamma)$ is called a metric l.c-space if $(X, d ; \Gamma)$ is a $c$-space such that open balls are $F$-sets and if any neighbourhood $\{x \in X \mid d(x, E)<r\}$ of any $F$-set $E \subset X$ is also an $F$-set [13]. 


\section{FiXed Point Theorems in KKM $L \Gamma$-SPACES}

From now on, all the topological spaces are assumed to be Hausdorff.

Let $X$ be an abstract convex space.

(I) For some $x_{0} \in X$, a map $T: X \multimap X$ is called a Mönch map if for a countable subset $B$ of $X, \bar{B}=\Gamma-\overline{c o}\left(\left\{x_{0}\right\} \cup T(B)\right)$ implies $\bar{B}$ is compact.

(II) For some precompact subset $C$ of $X$, a map $T: X \multimap X$ is called a Chandrabhan map if for a countable subset $B$ of $X, \bar{B}=\Gamma$ - $\overline{c o}(C \cup T(B))$ implies $\bar{B}$ is compact.

Clearly Mönch maps are Chandrabhan maps.

The following fixed point theorem was proved in [8].

Proposition 3.1. Let $(X ; \Gamma ; \mathscr{U})$ be a KKM LC-space, and let $T: X \multimap X$ be a compact upper semicontinuous map with closed $\Gamma$-convex values. Then $T$ has a fixed point.

The following fixed point theorem for upper semicontinuous multimaps is the main result of this paper.

Theorem 3.2. Let $(X ; \Gamma ; \mathscr{U})$ be a KKM L $\Gamma$-space with precompact polytopes. Let $C$ be a precompact subset of $X$, and let $T: X \multimap X$ be an upper semicontinuous multimap with compact $\Gamma$-convex values. Suppose that the following properties hold:

(1) for any compact subset $A$ of $X$, there exists a countable subset $B$ of $A$ with $\bar{B}=A$; and

(2) for a subset $A$ of $X, A=\Gamma-\overline{\mathrm{co}}(C \cup T(A))$ and $A=\bar{B}$ with some countable subset $B$ of $X$, implies $A$ is compact.

Then $T$ has a fixed point.

Proof. Put $K_{0}=\Gamma-\overline{\mathrm{co}}(C), K_{n+1}=\Gamma-\overline{\mathrm{co}}\left(C \cup T\left(K_{n}\right)\right)$ for $n=0,1,2, \cdots$, and $K=\bigcup_{n=0}^{\infty} K_{n}$. Вy induction, $K_{0} \subseteq K_{1} \subseteq \cdots \subseteq K_{n} \subseteq K_{n+1} \cdots$, and $K$ is $\Gamma$-convex since $K_{n}$ is $\Gamma$-convex for $n=$ $0,1,2, \cdots$.

Furthermore, we can show that $K=\Gamma-\overline{\mathrm{co}}(C \cup T(K))$. For each $n$,

$$
\Gamma-\overline{\mathrm{co}}\left(C \cup T\left(K_{n}\right)\right) \subseteq \Gamma-\overline{\mathrm{co}}(C \cup T(K)),
$$

so

$$
K=\bigcup_{n=0}^{\infty} \Gamma-\overline{\mathrm{co}}\left(C \cup T\left(K_{n}\right)\right) \subseteq \Gamma-\overline{\mathrm{co}}(C \cup T(K)) .
$$

On the other hand, $K$ is a closed $\Gamma$-convex set, which contains $C$ and $\bigcup_{n=0}^{\infty} T\left(K_{n}\right)=T(K)$. Hence

$$
\Gamma-\overline{\mathrm{co}}(C \cup T(K)) \subseteq K .
$$

By induction, we can show that $K_{n}$ is compact for $n=0,1,2, \cdots$, which is due to Lemma 2.2 and the assumption that $T$ is an upper semicontinuous multimap with compact values. Condition (1) implies that there exists a countable subset $B_{n}$ of $K_{n}$ with $\bar{B}_{n}=K_{n}$. Putting $B=\bigcup_{n=0}^{\infty} B_{n}$, we have $\bar{B}=K$, which is due to

$$
K=\bigcup_{n=0}^{\infty} K_{n}=\bigcup_{n=0}^{\infty} \overline{B_{n}}=\overline{\bigcup_{n=0}^{\infty} B_{n}}=\bar{B} .
$$

Condition (2) implies that $K$ is compact. Since $\left.T\right|_{K}: K \multimap K$ is a compact upper semicontinuous map with closed $\Gamma$-convex values, $\left.T\right|_{K}$ has a fixed point by Proposition 3.1. 
Remark 3.3. If $T$ also satisfies

$$
T(\bar{A}) \subset \overline{T(A)} \text { for every } A \subset X,
$$

then Chandrabhan maps satisfy the condition (2) in Theorem 3.2.

In fact, if $A=\Gamma-\overline{\mathrm{co}}(C \cup T(A))$, and $A=\bar{B}$ with some countable subset $B$ of $X$, then we can show that $\Gamma-\overline{\mathrm{co}}(C \cup T(A))=\Gamma-\overline{\mathrm{co}}(C \cup T(B))$, since $T(A)=T(\bar{B}) \subset \overline{T(B)} \subset \Gamma-\overline{\mathrm{co}}(C \cup T(B))$. This implies $\Gamma-\overline{\mathrm{co}}(C \cup T(A)) \subset \Gamma-\overline{\mathrm{co}}(C \cup T(B))$. Consequently, $\bar{B}=\Gamma-\overline{\mathrm{co}}(C \cup T(B))$, and the property of Chandrabhan maps guarantees that $\bar{B}=A$ is compact.

Since a lower semicontinuous multimap satisfies (3.1), we obtain the following theorem.

Theorem 3.4. Let $(X ; \Gamma ; \mathscr{U})$ be a KKM LC-space with precompact polytopes. Let $C$ be a precompact subset of $X$, and let $T: X \multimap X$ be a continuous Chandrabhan multimap with compact $\Gamma$-convex values. Suppose that, for any compact subset $A$ of $X$, there exists a countable subset $B$ of $A$ with $\bar{B}=A$. Then $T$ has a fixed point.

The following theorem is a fixed point theorem for a Chandrabhan type closed map.

Theorem 3.5. Let $(X ; \Gamma ; \mathscr{U})$ be a KKM LC-space with precompact polytopes. Let $C$ be a precompact subset of $X$, and let $T: X \multimap X$ be a closed multimap with $\Gamma$-convex values that satisfies the following properties:

(1) $T$ maps compact sets into precompact sets;

(2) for any compact subset $A$ of $X$, there exists a countable subset $B$ of $A$ with $\bar{B}=A$; and

(3) $A=\Gamma-\overline{\mathrm{co}}(C \cup T(A))$ with $A=\bar{B}$ and $B$ is countable, implies $A$ is compact.

Then $T$ has a fixed point.

Proof. Put $K_{0}=\Gamma-\overline{\mathrm{co}}(C), K_{n+1}=\Gamma-\overline{\mathrm{co}}\left(C \cup T\left(K_{n}\right)\right)$ for $n=0,1,2, \cdots$, and $K=\bigcup_{n=0}^{\infty} K_{n}$ as in the proof in Theorem 3.2. Then we can show that $K_{n}$ is compact for $n=0,1,2, \cdots$ by using Lemma 2.2 and condition (1). By the same argument of the proof in Theorem 3.2, we can show that $K$ is a nonempty compact $\Gamma$-convex subset of $X$ such that $T(K) \subset K$. Since $\left.T\right|_{K}: K \multimap K$ is closed, and $\overline{T(K)}$ is compact, $\left.T\right|_{K}$ is a upper semicontinuous map with closed $\Gamma$-convex values. Hence, $\left.T\right|_{K}$ has a fixed point by Proposition 3.1.

Corollary 3.6. Let $(X ; \Gamma ; \mathscr{U})$ be an LG-space with precompact polytopes. Let $C$ be a precompact subset of $X$, and let $T: X \multimap X$ be a closed multimap with $\Gamma$-convex values that satisfies the following properties:

(1) $T$ maps compact sets into precompact sets;

(2) for any compact subset $A$ of $X$, there exists a countable subset $B$ of $A$ with $\bar{B}=A$; and

(3) $A=\Gamma-\overline{\mathrm{co}}(C \cup T(A))$ with $A=\bar{B}$ and $B$ is countable, implies $A$ is compact.

Then $T$ has a fixed point.

Since a Banach space satisfies the condition (2) of Theorem 3.5, we obtain the following corollary, which generalizes the Theorem 3.1 in [5].

Corollary 3.7. Let $X$ be a Banach space. Let $C$ be a precompact subset of $X$, and let $T: X \multimap X$ be a closed multimap with convex values that satisfies the following properties:

(1) $T$ maps compact sets into precompact sets; and

(2) $A=\overline{\operatorname{co}(C \cup T(A))}$ with $A=\bar{B}$ and $B$ is countable, implies $A$ is compact.

Then $T$ has a fixed point. 


\section{Fixed Point Theorems on $\Phi$-SETS}

For a given abstract convex space $(X ; \Gamma)$, and a topological space $Y$, a map $H: Y \multimap X$ is called a $\Phi$-map if there exists a map $G: Y \multimap X$ such that

(i) for each $y \in Y, \operatorname{co}_{\Gamma} G(y) \subset H(y)$; and

(ii) $Y=\bigcup\left\{\operatorname{Int} G^{-}(x) \mid x \in X\right\}$.

In an abstract convex uniform space $(X ; \Gamma ; \mathscr{U})$, a subset $S$ of $X$ is called a $\Phi$-set if, for any entourage $U \in \mathscr{U}$, there exists a $\Phi$-map $H: S \multimap X$ such that $\mathrm{Gr} H \subset U$, where $\mathrm{Gr} H$ denotes the graph of $H$.

For an abstract convex uniform space $(X ; \Gamma ; \mathscr{U})$, if a subset $S$ of $X$ is a $\Phi$-set, then any subset of $S$ is a $\Phi$-set.

The following propositions were obtained in $[8,9]$.

Proposition 4.1. Let $(X ; \Gamma)$ be an abstract convex space. Let $C$ be a $\Gamma$-convex subset of $X$, and let $Z$ be a set. If $T \in \mathfrak{K}(X, Z)$, then $\left.T\right|_{C} \in \mathfrak{K}(C, Z)$.

Proposition 4.2. Let $(X ; \Gamma ; \mathscr{U})$ be an abstract convex uniform space, and let $T \in \mathfrak{K C}(X, X)$ be a compact closed map. If $\overline{T(X)}$ is a $\Phi$-set, then $T$ has a fixed point.

Theorem 4.3. Let $(X ; \Gamma ; \mathscr{U})$ be an $L \Gamma$-space with precompact polytopes. Let $C$ be a precompact subset of $X$, and let $T \in \mathfrak{K C}(X, X)$ be a closed multimap that satisfies the following properties:

(1) $T$ maps compact sets into precompact sets;

(2) for any compact subset $A$ of $X$, there exists a countable subset $B$ of $A$ with $\bar{B}=A$;

(3) $A=\Gamma-\overline{c o}(C \cup T(A))$ with $A=\bar{B}$ and $B$ is countable, implies $A$ is compact; and

(4) $\overline{T(X)}$ is a $\Phi$-set.

Then $T$ has a fixed point.

Proof. As the proof of Theorem 3.5, we can find a nonempty compact $\Gamma$-convex subset $K$ of $X$ such that $T(K) \subset K$, and $\left.T\right|_{K}: K \multimap K$ is compact closed. Since $\overline{T(X)}$ is a $\Phi$-set, so is $\overline{T(K)}$. By Proposition 4.1 and Proposition 4.2,T $\left.\right|_{K} \in \mathfrak{K C}(K, K)$, and $\left.T\right|_{K}$ has a fixed point.

Corollary 4.4. Let $(X ; \Gamma ; \mathscr{U})$ be an $L \Gamma$-space with precompact polytopes. Let $X$ be a $\Phi$-set, and let $T \in \mathfrak{K C}(X, X)$ be a closed multimap that satisfies the following properties:

(1) $T$ maps compact sets into precompact sets;

(2) for any compact subset $A$ of $X$, there exists a countable subset $B$ of $A$ with $\bar{B}=A$; and

(3) for $x_{0} \in X, A=\Gamma-\overline{\mathrm{co}}\left(\left\{x_{0}\right\} \cup T(A)\right)$ with $A=\bar{C}$ and $C$ is countable, implies $A$ is compact.

Then $T$ has a fixed point.

Corollary 4.4 remove san unnecessary condition of Theorem 2.4 in [7]. Note also that every singleton of an $\mathrm{L} \Gamma$-space $(X ; \Gamma ; \mathscr{U})$ is $\Gamma$-convex. Then, any subset of $X$ is a $\Phi$-set [9].

\section{Fixed Point Theorems on Metric Spaces}

Aronszajn and Panitchpakdi [16] called a metric space $(H, d)$ as a hyperconvex metric space if $\bigcap_{\alpha} B\left(x_{\alpha}, r_{\alpha}\right) \neq \emptyset$ for any collection $\left\{B\left(x_{\alpha}, r_{\alpha}\right)\right\}$ of closed balls in $H$ for which $d\left(x_{\alpha}, x_{\beta}\right) \leq$ $r_{\alpha}+r_{\beta}$.

For any hyperconvex metric space $(H, d)$, the following definition is due to Khamsi [17]:

(I) $\mathrm{BI}(A)=\bigcap\{B \subset H \mid B$ is a closed ball in $H$ such that $A \subset B\}$. 
(II) $A$ is called an admissible subset of $H$ if $A$ is an intersection of closed balls, that is, $A=\mathrm{BI}(A)$.

(III) A is called subadmissible if, for each $N \in\langle A\rangle, \mathrm{BI}(N) \subset A$.

Obviously, if $A$ is an admissible subset of $H$, then $A$ must be subadmissible.

The following useful result is due to Horvath [18].

Proposition 5.1. Any hyperconvex metric space $(H, d)$ is a complete metric l.c-space $(H, d ; \Gamma)$, where $\Gamma_{A}=\mathrm{BI}(\mathrm{A})$ for each $A \in\langle H\rangle$.

A $\Gamma$-convex subset of $(H, d ; \Gamma)$ is subadmissible and conversely.

Every polytope in a hyperconvex metric space is precompact, and a hyperconvex metric space satisfies condition (1) of Theorem 3.2, so we obtain the following fixed point theorems from Theorem 3.2 and Theorem 3.4 immediately.

Theorem 5.2. Let $(H, d ; \Gamma)$ be a hyperconvex metric space. Let $C$ be a precompact subset of $H$, and let $T: H \multimap H$ be a upper semicontinuous multimap with compact subadmissible values and satisfy that, for a subset $A$ of $X, A=\mathrm{BI}(C \cup T(A))$ and $A=\bar{B}$ with some countable subset $B$ of $X$ implies that $A$ is compact. Then $T$ has a fixed point.

Theorem 5.3. Let $(H, d ; \Gamma)$ be a hyperconvex metric space. Let $C$ be a precompact subset of $H$, and let $T: H \multimap H$ be a continuous Chandrabhan multimap with compact subadmissible values. Then $T$ has a fixed point.

Since every singleton of a hyperconvex metric space $(H, d ; \Gamma)$ is admissible, any subset of $H$ is a $\Phi$-set. Therefore we obtain the following theorem from Theorem 3.5 and Theorem 4.3 immediately.

Theorem 5.4. Let $(H, d ; \Gamma)$ be a hyperconvex metric space. Let $C$ be a precompact subset of $H$, and let $T: H \multimap H$ be a closed multimap that satisfies the following properties:

(1) $T$ maps compact sets into precompact sets; and

(2) $A=\mathrm{BI}(C \cup T(A))$ with $A=\bar{B}$ and $B$ is countable, implies $A$ is compact.

If $T$ has subadmissible values or $T \in \mathfrak{K C}(H, H)$, then $T$ has a fixed point.

\section{Acknowledgements}

The author was supported by the Sehan University Research Fund in 2021.

\section{REFERENCES}

[1] S. Park, Some general fixed point theorems on topological vector spaces, Appl. Set-Valued Anal. Optim. 1 (2019), 19-28.

[2] M. Darabi, Z. Soltani, J. Zafarani, Vector parametric quasi-equilibrium problems for the sum of two setvalued mappings, J. Nonlinear Var. Anal. 5 (2021), 267-279.

[3] C. Izuchukwu, Y. Shehu, Projection-type methods with alternating inertial steps for solving multivalued variational inequalities beyond monotonicity, J. Appl. Numer. Optim. 2 (2020), 249-277.

[4] H. Mönch, Boundary value problems for nonlinear ordinary differential equations of second order in Banach spaces, Nonlinear Anal. 4 (1980), 985-999.

[5] D. O'Regan, R. Precup, Fixed point theorems for set-valued maps and existence principles for integral inclusions, J. Math. Anal. Appl. 245 (2000), 594-612.

[6] B.C. Dhage, On Monch type multi-valued maps and fixed points, Appl. Math. Lett. 20 (2007), $622-628$. 
[7] A. Amini-Harandi, A. P. Farajzadeh, D. O’Regan, R. P. Agarwal, Fixed point theorems for condensing multimaps on abstract convex uniform spaces, Nonlinear Funct. Anal. Appl. 14 (2009), 109-120.

[8] S. Park, Fixed point theory of multimaps in abstract convex uniform spaces, Nonlinear Anal. 71 (2009), 2468-2480.

[9] S. Park, Elements of the KKM theory on abstract convex spaces, J. Korean Math. Soc. 45 (2008), 1-27.

[10] S. Park, H. Kim, Admissible classes of multifunctions on generalized convex spaces, Proc. Coll. Nat. Sci. Seoul Nat. Univ. 18 (1993), 1-21.

[11] S. Park, H. Kim, Coincidence theorems for admissible multifunctions on generalized convex spaces, J. Math. Anal. Appl. 197 (1996), 173-187.

[12] S. Park, H. Kim, Foundations of the KKM theory on generalized convex spaces, J. Math. Anal. Appl. 209 (1997), 551-571.

[13] C. D. Horvath, Contractibility and generalized convexity, J. Math. Anal. Appl. 156 (1991), 341-357.

[14] S. Park, Equilibrium existence theorems in KKM spaces, Nonlinear Anal. 69 (2008), 4352-4364.

[15] S. Park, Fixed point theorems in locally $G$-convex spaces, Equilibrium existence theorems in KKM spaces, Nonlinear Anal. 48 (2002), 869-879.

[16] N. Aronszajn, P. Panitchpakdi, Extensions of uniformly continuous transformations and hyperconvex metric spaces, Pacific J. Math. 6 (1956), 405-439.

[17] M.A. Khamsi, KKM and Ky Fan theorems in hyperconvex metric spaces, J. Math. Anal. Appl. 204 (1996), 298-306.

[18] C. D. Horvath, Extension and selection theorems in topological spaces with a generalized convexity structure, Ann. Fac. Sci. Toulouse 2 (1993), 253-269. 